\title{
Emergence of a Minor Clonal Dissemination of Klebsiella Pneumoniae Coproducing KPC-2 and IMP-4 Clone Among Pediatric Patients from China
}

\section{Ziyan Kong}

Xuzhou Medical University

Xuemei Liu

Children's Hospital of Nanjing Medical University

Chenxi Li

Xuzhou Medical University

Siyun Cheng

Xuzhou Medical University

Fei Xu

Children's Hospital of Nanjing Medical University

Bing Gu ( $\nabla$ gb20031129@163.com )

Department of Laboratory Medicine

\section{Research}

Keywords: Klebsiella pneumoniae, carbapenemase, KPC-2, ST11, clonal dissemination, children

Posted Date: October 28th, 2020

DOI: https://doi.org/10.21203/rs.3.rs-96881/v1

License: () (1) This work is licensed under a Creative Commons Attribution 4.0 International License.

Read Full License 


\section{Abstract}

Background: The continuous emergence of carbapenem-resistant Klebsiella pneumoniae (CRKP) has become a serious public health problem globally, especially for children, but data on CRKP infection in pediatric patients are limited. This study aimed to identify epidemiological and molecular patterns of CRKP among pediatric patients in Jiangsu province, China.

Methods: CRKP were consecutively collected from the Children's Hospital of Nanjing Medical University in China from July 2018 to May 2019. Then CRKP strains were performed for further study: antimicrobial susceptibility testing, drug resistance determinants screening and homology analysis.

Results: Overall, b/a $a_{\mathrm{KPC}-2}(79.8 \%)$ was the predominant carbapenemase gene, followed by b/a $a_{\mathrm{NDM}}$ ${ }_{1}(14.9 \%), b / a_{\mid \mathrm{MP}-4}(5.3 \%)$ and $b / a_{\mathrm{NDM}-5}(4.3 \%)$. Notably, two isolates coharbored $b / a_{\mathrm{KPC}-2}$ and $b / a_{\mathrm{IMP}-4}$, and two isolates coharbored $b / a_{\mathrm{KPC}-2}$ and $b / a_{\mathrm{NDM}-5}$. MLST analysis revealed that fourteen distinct sequence types (STs) were identified, of which ST11 was the most common sequence type identified. Moreover, two novel STs, ST4854 and ST4855, were detected in this study. PFGE revealed that a predominant cluster consisting of KPC-2-producing CRKP ST11 clone isolates was identified and was distributed mainly in the pediatric intensive care unit (PICU) and cardiac intensive care unit (CCU). Moreover, this is the first report to identify the minor clonal dissemination of CRKP ST716 coproducing KPC-2 and IMP-4 and CRKP ST1140 producing NDM-5.

Conclusions: Clonal dissemination of KPC-2-producing CRKP ST11 was observed in multiple departments. Moreover, two novel STs (ST4854 and ST4855) were identified, which indicates increased diversity of CRKP strains. To our knowledge, this is the first report that identified a minor clonal dissemination of Klebsiella pneumoniae coproducing KPC-2 and IMP-4 clones among children, which represents a significant health risk to pediatric patients. Active surveillance and effective control measures are urgently needed to prevent further transmission of these strains among children.

\section{Backgroud}

The emergence of carbapenem-resistant Klebsiella pneumoniae (CRKP) has become a serious global public health problem that spreads among our most vulnerable population, children (Logan, 2012). In 2017, the World Health Organization (WHO) published its first list of antibiotic-resistant "priority pathogens" - a catalog of 12 families of bacteria that pose the greatest threat to human health, and CRKP was listed as a critical priority pathogen (WHO, 2017). According to data from the China Antimicrobial Surveillance Network (CHINET) for 2005-2017, the prevalence of CRKP in children dramatically increased from $2.2 \%$ (5.0\% in adults) to $25.4 \%$ (21.2\% in adults) (Guo et al., 2018). The production of carbapenemase is the major mechanism of CRKP strain resistance to carbapenem. Carbapenemases reported worldwide mainly include class A enzyme Klebsiella pneumoniae carbapenemase (KPC), class B enzyme New Delhi metallo- $\beta$-lactamase (NDM) and class D enzyme oxacillinase type 48 (OXA-48). In 
China, previous studies revealed that the main carbapenemase of adults was KPC-2, while the predominant carbapenemase of pediatric patients was NDM-1 (Zhang et al., 2018; Dong et al., 2017).

Children, as naturally vulnerable groups, have limited choice of antibiotics due to side effects. In addition, carbapenem resistance genes and other resistance genes are often detected in CRKP strains simultaneously, which increases the difficulty of anti-infection treatment in children (Munoz- Price et al., 2009). Previous studies showed high morbidity and mortality in pediatric patients infected with CRKP (Logan, 2012). Therefore, more information about the molecular characteristics of CRKP is needed to effectively control the spread of CRKP and prevent outbreaks in pediatric patients in the future. There are more previous studies focused on CRKP in adults than in children, while epidemiological data on children's CRKP are relatively limited. Therefore, we aimed to investigate the resistance determinants, antibiotic resistance characteristics, and genetic relatedness among CRKP isolates from pediatric patients at the Children's Hospital of Nanjing Medical University in Jiangsu Province, China.

\section{Methods}

\section{Collection of bacterial strains}

Consecutive nonduplicated strains of CRKP were prospectively collected from July 2018 to May 2019 from inpatients who were admitted to Children's Hospital of Nanjing Medical University, which is one of the largest Children's hospitals in Jiangsu, China. There are approximately1400 beds in the hospital with an estimated population of two million patient visits per year. Strain identification was performed by matrix-assisted laser desorption/ionization time-of-flight mass spectrometry. In vitro antimicrobial susceptibility testing of isolates was analyzed with a VITEK-2 compact system (bioMérieux, Marcyl'Étoile, France). The electronic medical records of the children, including patient age, sex, ward, underlying diseases and specimen source, were retrospectively reviewed.

\section{Antimicrobial susceptibility testing}

The minimum inhibitory concentrations (MICs) of imipenem, meropenem, cefazolin, cefuroxime, ceftazidime, cefepime, piperacillin-tazobactam, aztreonam, amikacin, ciprofloxacin, colistin, tigecycline, and sulfamethoxazole were detected by the broth microdilution method. Susceptibility breakpoints were interpreted in accordance with the Clinical and Laboratory Standards Institute (CLSI), except for tigecycline and colistin, which were interpreted based on the European Committee for Antimicrobial Susceptibility Testing (EUCAST) criteria (CLSI, 2018; Kahlmeter et al., 2016).

\section{Detection of resistance determinant}

PCR using the primers was conducted as previously described and used to detect carbapenemase genes $\left(b / a_{\mathrm{KPC}}, b / a_{\mathrm{NDM}}, b l_{\mathrm{aIMP}}, b / a_{\mathrm{VIM}}, b / a_{\mathrm{OXA}-48}\right)$, common extended-spectrum $\beta$-lactamase (ESBL) genes (b/a $a_{\mathrm{CTX}-\mathrm{M}}, b / a_{\mathrm{SHV}}$ and $\left.b / a_{\mathrm{TEM}}\right)$ and plasmid-encoding AmpC genes (b/a $\left.a_{\mathrm{DHA}}, b / a_{\mathrm{CIT}}, b / a_{\mathrm{MOX}}, b / a_{\mathrm{EBC}}, b / a_{\mathrm{FOX}}\right)$ (Endimiani et al., 2008; Yang et al., 2012; Senda et al., 1996; Tsakris et al., 2000; Poirel et al., 2004; Yu et 
al., 2007; Pérez-Pérez et al., 2002). The amplicons were screened by electrophoresis on a $1.5 \%$ agarose gel. The positive amplicons were sequenced using Sanger sequencing, and the entire sequences obtained were compared to the reported sequences in GenBank using the BLAST algorithm (http://www.ncbi.nlm.nih.gov/blast/).

\section{Molecular typing}

Multilocus sequence typing (MLST) was performed for genotyping according to the protocol described previously on the MLST website of the Pasteur Institute (http://bigsdb.pasteur.fr/klebsiella/klebsiella.html). The sequences of seven housekeeping genes (infB, pgi, mdh, phoE, gapA, tonB and rpoB) were compared with those in the Klebsiella pneumoniae MLST database. A minimum spanning tree of 94 Klebsiella pneumoniae strains was constructed in BioNumerics software version 7.6.

The clonal relationships of Klebsiella pneumoniae isolates were determined by pulsed-field gel electrophoresis (PFGE). PFGE was performed according to the protocol established by the Centers for Disease Control and Prevention (Atlanta, GA). The PFGE patterns were also compared using BioNumerics software version 7.6 , with a cutoff at $80 \%$ similarity to indicate identical PFGE types.

\section{Results}

\section{Clinical characteristics of CRKP isolates}

A total of 94 nonrepetitive Klebsiella pneumoniae strains that exhibited resistance to carbapenems were obtained from the Children's Hospital of Nanjing Medical University from July 2018 to May 2019. The clinical and epidemiological characteristics of these isolates are summarized in Table 1. Children who were colonized or infected with CRKP had a median age of 4 months (interquartile range, 2-12 months), and the male-to-female ratio was 1.7. The isolated CRKP strains were collected from clinical specimens, which included sputum $(72.3 \%, 68 / 94)$, urine $(9.6 \%, 9 / 94)$, blood $(8.5 \%, 8 / 94)$, and other samples $(9.6 \%$, 9/94). The CRKP strains were isolated from pediatric patients in 16 different wards but were primarily from PICU $(26.6 \%, 25 / 94)$, pneumology department $(13.9 \%, 13 / 94)$ and CCU $(13.9 \%, 13 / 94)$. From electronic medical records, the most common diseases in patients were pneumonia $(62.8 \%, 59 / 94)$ and sepsis $(18.1 \%, 17 / 94)$. 
Table 1

Clinical characteristics of the CRKP strains

\begin{tabular}{|ll|}
\hline Characteristics & $\mathbf{n}=94$ \\
\hline Age in months(median) & 4 \\
\hline Male gender & $59(62.8 \%)$ \\
\hline Specimen & \\
\hline Sputum & $68(72.3 \%)$ \\
\hline Urine & $9(9.6 \%)$ \\
\hline Blood & $8(8.5 \%)$ \\
\hline Others & $9(9.6 \%)$ \\
\hline Wards & \\
\hline PICU & $25(26.6 \%)$ \\
\hline Pneumology department & $13(13.9 \%)$ \\
\hline CCU & $13(13.9 \%)$ \\
\hline Neonatal medicine & $12(12.8 \%)$ \\
\hline Others & $31(67.0 \%)$ \\
\hline Diseases & \\
\hline Pneumonia & $59(62.8 \%)$ \\
\hline Sepsis & $3(8.5 \%)$ \\
\hline Cerebral disease & $3(3.2 \%)$ \\
\hline Urinary tract infection & $2(2.1 \%)$ \\
\hline Underlying conditions & $2(2.1 \%)$ \\
\hline Chronic Heart Disease & $1(18.1 \%)$ \\
\hline Neonatal Respiratory Distress Syndrome & \\
\hline Gastroenteritis & \\
\hline Immunodeficiency & \\
\hline outcome & \\
\hline Cure & \\
\hline Death & \\
\hline
\end{tabular}




\section{Antibiotic susceptibility testing}

The results of the antimicrobial susceptibility testing of the 94 CRKP strains are shown in Table 2. All 94 clinical isolates were observed as multidrug resistant bacteria and exhibited resistance to meropenem and cephalosporins and to enzyme inhibitors. The rates of susceptibility to imipenem, aztreonam, ciprofloxacin, amikacin and sulfamethoxazole were $1.1 \%, 4.3 \%, 18.1 \%, 43.6 \%$ and $85.1 \%$, respectively. Additionally, the CRKP strains showed a high susceptibility rate to tigecycline and colistin $(100.0 \%)$.

Table 2

Antimicrobial susceptibility patterns of CRKP strains, \% (n)

\begin{tabular}{|llll|}
\hline Antibioics & Susceptible & Intermediate & Resistant \\
\hline imipenem & $1.1 \%(1 / 94)$ & $0.0(0)$ & $98.9(93)$ \\
\hline meropenem & $0.0(0)$ & $0.0(0)$ & $100.0(94)$ \\
\hline piperacillin-tazobactam & $0.0(0)$ & $0.0(0)$ & $100.0(94)$ \\
\hline cefazolin & $0.0(0)$ & $0.0(0)$ & $100.0(94)$ \\
\hline cefuroxime & $0.0(0)$ & $0.0(0)$ & $100.0(94)$ \\
\hline ceftazidime & $0.0(0)$ & $0.0(0)$ & $100.0(94)$ \\
\hline cefepime & $0.0(0)$ & $0.0(0)$ & $100.0(94)$ \\
\hline aztreonam & $4.3(0)$ & $0.0(0)$ & $95.7(90)$ \\
\hline ciprofloxacin & $18.1(17)$ & $2.1 \%(2)$ & $79.8(75)$ \\
\hline amikacin & $43.6(41)$ & $0.0(0)$ & $56.4(53)$ \\
\hline sulfamethoxazole & $85.1(80)$ & $0.0(0)$ & $14.9(14)$ \\
\hline tigecycline & $100.0(94)$ & $0.0(0)$ & $0.0(0)$ \\
\hline colistin & $100.0(94)$ & $0.0(0)$ & $0.0(0)$ \\
\hline
\end{tabular}

\section{Detection of resistance determinants}

All 94 isolates carried the carbapenemase genes, and $b / a_{\mathrm{KPC}-2}(79.8 \%, 75 / 94)$ was predominantly detected, followed by bla $a_{\mathrm{NDM}-1}(14.9 \%, 14 / 94)$, bla $a_{\mathrm{MP}-4}(5.3 \%, 5 / 94)$ and $b / a_{\mathrm{NDM}-5}(4.3 \%, 4 / 94)$.

Notably, two strains coharboring $b / a_{\mathrm{KPC}-2}$ and $b / a_{\mathrm{IMP}-4}$ were obtained from two children in the PICU who underwent mechanical ventilation and died of severe lung infection or septic shock. In addition, two strains coharboring $b / a_{\mathrm{KPC}-2}$ and $b / a_{\mathrm{NDM}-5}$ were also isolated from two children in the PICU. 
All isolates were successfully identified with ESBL genes. Bla $a_{S H V}$ was the most prevalent among the ESBL genes. The common subtypes of $b / a_{\mathrm{SHV}}$ in these strains were bla $a_{\mathrm{SHV}-11}(46.8 \%, 44 / 94), b / a_{\mathrm{SHV}-12}(24.5 \%$, 23/94), bla $a_{\mathrm{SHV}-2 \mathrm{a}}(7.4 \%, 7 / 94)$, bla $\mathrm{SHV}_{\mathrm{SH}-1}(6.4 \%, 6 / 94), b_{\mathrm{SHV}-27}(2.1 \%, 2 / 94), \mathrm{bla}_{\mathrm{SHV}-26}(1.1 \%, 1 / 94)$, $b / a_{\mathrm{SHV}-28}(1.1 \%, 1 / 94)$ and $b / a_{\mathrm{SHV}-187}(1.1 \%, 1 / 94)$. Other ESBL genes were $b / a_{\mathrm{TEM}-1}(\mathrm{n}=68), b / a_{\mathrm{CTX}-\mathrm{M}-15}$ $(\mathrm{n}=13), b / a_{\mathrm{CTX}-\mathrm{M}-65}(\mathrm{n}=6), b / a_{\mathrm{CTX}-\mathrm{M}-27}(\mathrm{n}=1)$ and bla $a_{\mathrm{CTX}-\mathrm{M}-14}(\mathrm{n}=1)$. The $b / a_{\mathrm{DHA}-1}$ gene, which is an AmpC $\beta$-lactamase gene, was detected in one strain.

\section{Molecular typing}

Among the 94 CRKP strains, a total of 14 different sequence types (STs) (12 previously described STs and 2 novel STs) were identified. ST11 $(75.5 \%, 71 / 94)$ was the most common type, followed by ST76 $(8.5 \%, 8 / 94)$ and other uncommon STs that were identified in one or two strains each. Furthermore, two new STs (ST4854 and ST4855) represented two novel allelic profiles of seven housekeeping genes. The distributions of carbapenemases among different STs are shown in Fig. 1. This study showed that most KPC-2 producers $(94.7 \%, 71 / 75)$ belonged to ST11, whereas NDM-1 producers belonged to many STs such as ST76 $(n=7)$, ST20 $(n=2)$, ST17 $(n=1)$, ST35 $(n=1)$, ST193 $(n=1)$, ST690 $(n=1)$, and ST4854 $(n=1)$. Strains carrying the IMP-4 gene belonged to ST716 $(n=2)$, ST34 $(n=1)$, ST76 $(n=1)$, and ST307 $(n=1)$. The NDM-5 gene belonged to ST1140 $(n=2), \operatorname{ST11}(n=1)$ and ST4855 $(n=1)$. Additionally, two isolates coharboring KPC-2 and IMP-4 were typed as ST716, and two strains coharboring KPC-2 and NDM-5 were typed as ST11 and ST4855, respectively.

PFGE patterns of Xbal-digested genomic DNA of 94 CRKP isolates revealed 12 different clusters. PFGE profiles of 71 ST11 CRKP isolates were grouped into 3 different PFGE clusters. The predominant cluster consisting of 63 KPC-2-producing CRKP ST11 clone strains was identified, indicating clone dissemination of those isolates mainly from the PICU $(n=16)$, CCU $(n=11)$ and pneumology department $(n=9)$ (Fig. 2). Furthermore, the PFGE profiles of the other 23 non-ST11 CRKP isolates were grouped into nine different PFGE clusters (Fig. 3). Among them, 7 NDM-1-producing CRKP ST76 clones displayed the same PFGE profiles, which revealed clonal dissemination of these strains from the pneumology department $(n=3)$, neonatal medicine department $(n=3)$, and infectious disease department $(n=1)$. Two isolates coharboring KPC-2 and IMP-4 belonged to ST716 from the PICU and shared highly similar PFGE patterns. In addition, two NDM-5-producing ST1140 clones and two NDM-1-producing CRKP ST20 clones from the surgical intensive care unit (SICU) shared the same PFGE profiles. In general, the clonal dissemination of CRKP was identified in multiple departments, with the intensive care unit being the most common.

\section{Discussion}

CRKP is an emerging problem that spreads among our most vulnerable population, children. Previous studies have mostly focused on adults, and molecular epidemiological data on children are limited. The 
aim of the present study was to describe the microbial resistance characteristics and epidemiological clinical characteristics of CRKP, which may help to prevent CRKP from becoming an epidemic in children. To the best of our knowledge, this is the first report of a small clonal spread of Klebsiella pneumoniae ST716 coproducing KPC-2 and IMP-4 and Klebsiella pneumoniae and ST1140 producing NDM-5 among pediatric patients.

In this study, all 94 CRKPs were multidrug resistant bacteria, but the resistance rate of strains to sulfamethoxazole, tigecycline and colistin was low, which may be due to their side effects; thus, these antibiotics are rarely used in pediatric patients in China. A previous study revealed that colistin in combination with other antimicrobial agents resulted in relatively low nephrotoxicity and a favorable outcome in $>70 \%$ of pediatric patients with infections due to carbapenem-resistant bacteria (Antachopoulos et al., 2017). It is worth noting that with the continuous use of colistin in clinical practice, Klebsiella pneumoniae has been reported to be resistant to colistin, and its resistance mechanism is different from previous chromosomal mutations but is caused by the colistin resistance gene (mcr- 1 ) carried by plasmids (Quan et al., 2017). With the limited choice of antimicrobial agents in children, there is an urgent need to take effective infection control measures and strengthen continuous surveillance to prevent further dissemination of CRKPs among pediatric patients.

KPC was previously described as the most common type of carbapenemase in Klebsiella pneumoniae strains in adults (Wang et al., 2018), which is consistent with the current results that the $94(79.8 \%)$ CRKPs collected from children mainly produced the KPC-2 enzyme. In addition, homology analysis showed that KPC-2-producing CRKP ST11 clones were widely spread in multiple departments, mainly in the PICU and CCU. ST11 was the dominant clone of KPC-2-producing CRKP isolates in China. A previous study revealed that ST11 Klebsiella pneumoniae showed a pandrug-resistant phenotype with a high prevalence of virulence factors favoring the binding, biofilm formation, colonization and escape from phagocytosis, which can make clones of this pathogen successfully spread worldwide (Andrade et al., 2014). In this study, NDM-1-producing Klebsiella pneumoniae were identified, accounting for $14.9 \%$ of the CRKPs collected. A five-year surveillance of CRKP strains in China revealed that NDM-1 enzymes gradually became the most prevalent type of carbapenemase in children (Dong et al., 2017). The current findings revealed that ST76 and ST20 Klebsiella pneumoniae cause clonal dissemination of NDM-1producing Klebsiella pneumoniae in our hospital. It has been reported that NDM-1-producing Klebsiella pneumoniae ST76 caused an infection outbreak in the neonatal unit in Shanghai and that NDM-1producing Klebsiella pneumoniae ST20 caused an infection outbreak in the neonatal unit in Shandong Province, suggesting that ST76 and ST20 are potentially high-risk clones that need more attention (Zhu et al., 2016; Jin et al., 2015). Moreover, NDM-1-producing Klebsiella pneumoniae had different clonal backgrounds, and NDM-1 was also observed in ST17, ST35, ST690 and ST4854 isolates in our study. To our knowledge, ST690 and ST4854 NDM-1-producing Klebsiella pneumoniae have never been reported globally, indicating that the diversity of NDM-1-producing Klebsiella pneumoniae is increasing. The NDM5 enzyme, first identified in the Escherichia coli strain in the UK, appeared to show increased hydrolytic activity to carbapenems and extended-spectrum cephalosporins (Hornsey et al., 2011; Rahman et al., 2014). Notably, our previous research found that the NDM-5 enzyme was associated with nosocomial 
outbreaks of ST337 Klebsiella pneumoniae in a neonatal unit in Jiangsu Province (Kong et al., 2019). Our current results show that the first minor clonal spread of $b / a_{\mathrm{NDM}-5}$-harboring ST1140 clone strains was identified in the SICU at the Children's Hospital of Nanjing Medical University, revealing rapid evolution of CRKP strains among pediatric patients.

Notably, two ST716 Klebsiella pneumoniae strains coharboring $b / a_{\mathrm{KPC}-2}$ and $b / a_{\mathrm{IMP}-4}$ and a novel Klebsiella pneumoniae sequence type 4855 carrying both $b / a_{\mathrm{KPC}-2}$ and $b / a_{\mathrm{NDM}-5}$ were identified. The KPC enzyme has the ability to hydrolyze monobactam antibiotics but can be inhibited by some $\beta$-lactamase inhibitors, such as avibactam. However, avibactam is inactive against metallo- $\beta$-lactamase (MBL) producers, including NDM and IMP, and monobactam antibiotics are active against MBL producers (Chiotos et al., 2020). Therefore, the synergistic effects of different carbapenemase classes may lead to higher levels of resistance to carbapenems and other antimicrobials. Due to the limited choice of antibiotics in children, the emergence of these strains brings great challenges to clinical treatment.

Up to now, only one KPC-2 and IMP-4 co-producing CRKP isolate and one strain harbored $b / a_{\mathrm{KPC}-2}$ and $b / a_{\text {NDM-5 }}$ have been identified from children (Tian et al., 2018; Zhou et al., 2020). To our knowledge, this is the first report of a minor clonal dissemination of Klebsiella pneumoniae coproducing KPC-2 and IMP-4 carbapenemases in two PICU patients. Something of concern is that these two pediatric patients died of severe lung infection, which suggests that the Klebsiella pneumoniae isolates coproducing two carbapenemases may pose a significant health risk to pediatric patients. A previous study revealed that Klebsiella pneumoniae coproducing the KPC-2 and NDM-1 enzymes are highly stable, have interhost transmission capacity, and are resistant to the newly marketed ceftazidime-avibactam (Gao et al., 2020). Therefore, effective infection control measures are urgently required to prevent further spread of the strains coproducing two carbapenemases among children.

There are some limitations in this study. First, it was performed at a third-grade children's hospital in Jiangsu province, and molecular epidemiology and drug-resistance mechanisms of CRKP strains in our children may not be generalizable to children patients throughout our country. In addition, the clinical characteristics of patients with CRKP isolates were briefly reviewed in our study, and we did not identify independent risk factors for CRKP strains infections due to a lot of related research has been performed, and our study focused mainly on clinical molecular epidemiology of carbapenem-resistant Klebsiella pneumoniae among pediatric patients.

\section{Conclusions}

In summary, the production of KPC-2 was the main mechanism of carbapenem resistance among pediatric patients in Nanjing, Jiangsu Province. Clonal dissemination of KPC-2-producing Klebsiella pneumoniae ST11 isolates was observed in multiple departments, with PICU and CCU being the most common, showing extensive cross-transmission of CRKP strains among high-risk wards. This was the first report of a minor clonal dissemination of Klebsiella pneumoniae coproducing KPC-2 and IMP-4 carbapenemases in children, and the detection of the Klebsiella pneumoniae isolates coproducing two 
carbapenemases represents a significant health risk to pediatric patients. Therefore, active surveillance and strict infection control measures are urgently needed to prevent Klebsiella pneumoniae strains coproducing two or more carbapenemases from becoming epidemic among children in the future.

\section{Abbreviations}

Carbapenem-resistant Klebsiella pneumoniae (CRKP), pediatric intensive care unit (PICU), cardiac intensive care unit (CCU), World Health Organization (WHO), China Antimicrobial Surveillance Network (CHINET), Klebsiella pneumoniae carbapenemase (KPC), New Delhi metallo- $\beta$-lactamase (NDM), oxacillinase type 48 (OXA-48), minimum inhibitory concentrations (MICs), Clinical and Laboratory Standards Institute (CLSI), European Committee for Antimicrobial Susceptibility Testing (EUCAST), extended-spectrum $\beta$-lactamase (ESBL), multilocus sequence typing (MLST), pulsed-field gel electrophoresis (PFGE), metallo- $\beta$-lactamase (MBL).

\section{Declarations}

\section{Acknowledgments}

We thank the team of curators of the Institute Pasteur MLST for curating the data and making them publicly available at (http://bigsdb.pasteur.fr/klebsiella/klebsiella.html).

\section{Funding}

This research was supported by the National Natural Science Foundation of China (16611840).

\section{Availability of Data}

Data is with first and corresponding author.

\section{Authors' Contributions}

The laboratory measurements were carried by ZK, XL and CL. BG and FX participated in experimental design and manuscript revision. $\mathrm{ZK}, \mathrm{XL}, \mathrm{CL}$ and $\mathrm{SC}$ contributed toward data analysis. All authors read and approved the final manuscript.

\section{Competing interests}

The authors declare that they have no competing interests 


\section{Consent for publication}

Not applicable.

\section{Ethical approval}

The Clinical Research Ethics Committee of the Children's Hospital of Nanjing Medical University approved the study (201907222-1), as all samples collected in this work were initially used to diagnose patient care without increasing the patient's medical costs and suffering.

\section{References}

1. Andrade LN, Vitali L, Gaspar GG, Bellissimo-Rodrigues F, Martinez R, Darini AL. Expansion and evolution of a virulent, extensively drug-resistant (polymyxin B-resistant), QnrS1-, CTX-M-2-, and KPC2-producing Klebsiella pneumoniae ST11 international high-risk clone. J Clin Microbiol. 2014;52(7):2530-5.

2. Antachopoulos $C$, losifidis E. Colistin use in neonates and children with infections due to carbapenem-resistant bacteria. Pediatr Infect Dis J. 2017;36(9):905-7.

3. Chiotos K, Hayes M, Gerber JS, Tamma PD. Treatment of Carbapenem-Resistant Enterobacteriaceae Infections in Children. Journal of the Pediatric Infectious Diseases Society. 2020;9(1):56-66.

4. Clinical and Laboratory Standards Institute. Performance Standards for Antimicrobial Susceptibility Testing. M100 -S28. Wayne: CLSl; 2018.

5. Dong F, Lu J, Wang Y, Shi J, Zhen JH, Chu P, et al. A five-year surveillance of carbapenemaseproducing Klebsiella pneumoniae in a pediatric hospital in China reveals increased predominance of NDM-1. Biomedical environmental sciences. 2017;30(8):562-9.

6. Endimiani A, Carias LL, Hujer AM, Bethel CR, Hujer KM, Perez F, et al. Presence of plasmid-mediated quinolone resistance in Klebsiella pneumoniae isolates possessing blaKPC in the United States. Antimicrob Agents Chemother. 2008;52(7):2680-2.

7. Gao H, Liu Y, Wang R, Wang Q, Jin L, Wang H. The transferability and evolution of NDM-1 and KPC-2 co-producing Klebsiella pneumoniae from clinical settings. EBioMedicine. 2020;51:102599.

8. Guo Y, Hu FP, Zhu DM, Wang CQ, Wang AM, Zhang H, et al. Antimicrobial resistance changes of carbapenem-resistant strains isolated from children. Zhonghua Er Ke Za Zhi. 2018;56(12):907-14.

9. Hornsey M, Phee L, Wareham DW. A novel variant, NDM-5, of the New Delhi metallo- $\beta$-lactamase in a multidrug-resistant Escherichia coli ST648 isolate recovered from a patient in the United Kingdom. Antimicrob Agents Chemother. 2011;55(12):5952-4.

10. Jin Y, Shao C, Li J, Fan H, Bai Y, Wang Y. Outbreak of multidrug resistant NDM-1-producing Klebsiella pneumoniae from a neonatal unit in Shandong Province, China. PLoS One. 2015;10(3):e0119571. 
11. Kahlmeter G, Brown D, Goldstein F, et al. European Committee on Antimicrobial Susceptibility Testing (EUCAST) technical notes on antimicrobial susceptibility testing. Clin Microbiol Infect. 2006;12(6):501-3.

12. Kong Z, Cai R, Cheng C, Zhang C, Kang H, Ma P, et al. First reported nosocomial outbreak of NDM-5producing Klebsiella pneumoniae in a neonatal unit in China. Infection Drug Resistance. 2019;12:3557-66.

13. Logan LK. Carbapenem-resistant Enterobacteriaceae: an emerging problem in children. Clinical infectious diseases. 2012;55(6):852-9.

14. Munoz-Price LS, Quinn JP. The spread of Klebsiella pneumoniae carbapenemases: a tale of strains, plasmids, and transposons. Clin Infect Dis. 2009;49(11):1739-41.

15. Pérez-Pérez FJ, Hanson ND. Detection of plasmid-mediated AmpC $\beta$-lactamase genes in clinical isolates by using multiplex PCR. J Clin Microbiol. 2002;40(6):2153-62.

16. Poirel L, Héritier C, Tolün V, Nordmann P. Emergence of oxacillinase-mediated resistance to imipenem in Klebsiella pneumoniae. Antimicrob Agents Chemother. 2004;48(1):15-22.

17. Quan J, Li X, Chen Y, Jiang Y, Zhou Z, Zhang H, et al. Prevalence of mcr-1 in Escherichia coli and Klebsiella pneumoniae recovered from bloodstream infections in China: a multicentre longitudinal study. Lancet Infect Dis. 2017;17(4):400-10.

18. Rahman M, Shukla SK, Prasad KN, Ovejero CM, Pati BK, Tripathi A, et al. Prevalence and molecular characterisation of New Delhi metallo- $\beta-$ lactamases NDM-1, NDM-5, NDM-6 and NDM-7 in multidrugresistant Enterobacteriaceae from India. Int J Antimicrob Agents. 2014;44(1):30-7.

19. Senda K, Arakawa Y, Ichiyama S, Nakashima K, Ito H, Ohsuka S, et al. PCR detection of metallo-betalactamase gene (blaIMP) in gram-negative rods resistant to broad-spectrum beta-lactams. J Clin Microbiol. 1996;34(12):2909-13.

20. Tian D, Pan F, Wang C, Sun Y, Zhang H. Resistance phenotype and clinical molecular epidemiology of carbapenem-resistant Klebsiella pneumoniae among pediatric patients in Shanghai. Infection and drug resistance. 2018;11: 1935.

21. Tsakris A, Pournaras S, Woodford N, Palepou MF, Babini GS, Douboyas J, et al. Outbreak of infections caused by Pseudomonas aeruginosa producing VIM-1 carbapenemase in Greece. J Clin Microbiol. 2000;38(3):1290-2.

22. Wang Q, Wang X, Wang J, Ouyang P, Jin C, Wang R, et al. Phenotypic and genotypic characterization of carbapenem-resistant Enterobacteriaceae: data from a longitudinal large-scale CRE study in China (2012-2016). Clin Infect Dis. 2018;67(suppl_2):196-205.

23. WHO. WHO publishes list of bacteria for which new antibiotics are urgently needed. https://. Accessed 27 February 2017.

24. Yang J, Chen Y, Jia X, Luo Y, Song Q, Zhao W, et al. Dissemination and characterization of NDM-1producing Acinetobacter pittii in an intensive care unit in China. Clin Microbiol Infect. 2012;18(12):E506-E13. 
25. Yu Y, Ji S, Chen Y, Zhou W, Wei Z, Li L, et al. Resistance of strains producing extended-spectrum $\beta$ lactamases and genotype distribution in China. J Infect. 2007;54(1):53-7.

26. Zhang Y, Wang Q, Yin Y, Chen H, Jin L, Gu B, et al. Epidemiology of carbapenem-resistant Enterobacteriaceae infections: report from China CRE Network. Antimicrobial Agents \& Chemotherapy, AAC. 2018;62(2): 01882-17.

27. Zhou J, Yang J, Hu F, Gao K, Sun J, Yang J. Clinical and Molecular Epidemiologic Characteristics of Ceftazidime/Avibactam-Resistant Carbapenem-Resistant Klebsiella pneumoniae in a neonatal Intensive Care Unit in China. Infection Drug Resistance. 2020;13:2571.

28. Zhu J, Sun L, Ding B, Yang Y, Xu X, Liu W, et al. Outbreak of NDM-1-producing Klebsiella pneumoniae ST76 and ST37 isolates in neonates. European journal of clinical microbiology infectious diseases. 2016;35(4):611-8.

\section{Figures}




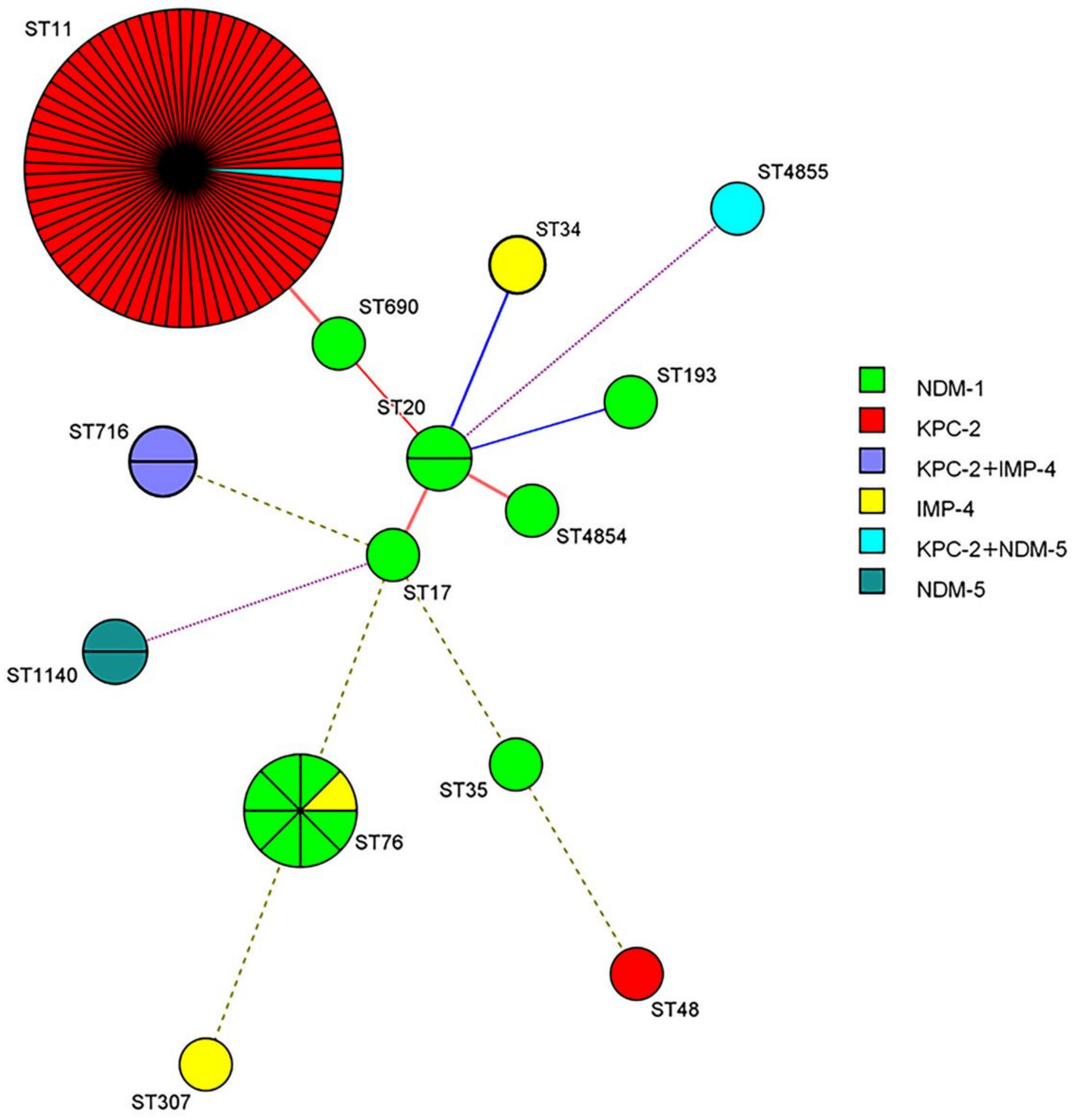

Figure 1

Minimum spanning trees of 94 CRKP isolates. 


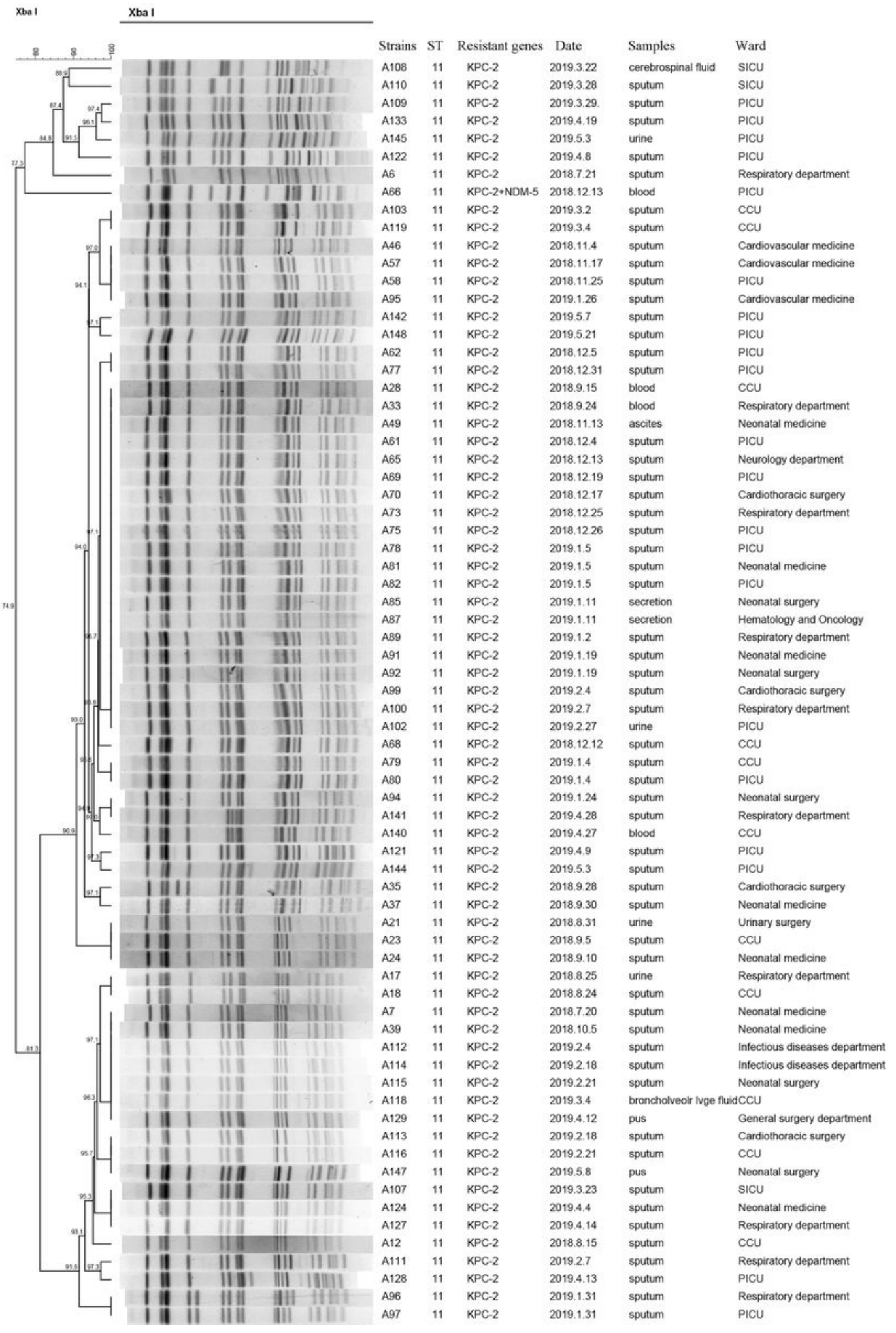

Figure 2

Dendrogram of PFGE profiles of 71 CRKP ST 11 isolates. The UPGMA algorithm was performed to construct a dendrogram based on the dice similarity coefficient. Strains were classified as the same clone cluster when their dice similarity index was $\geq 80 \%$. 


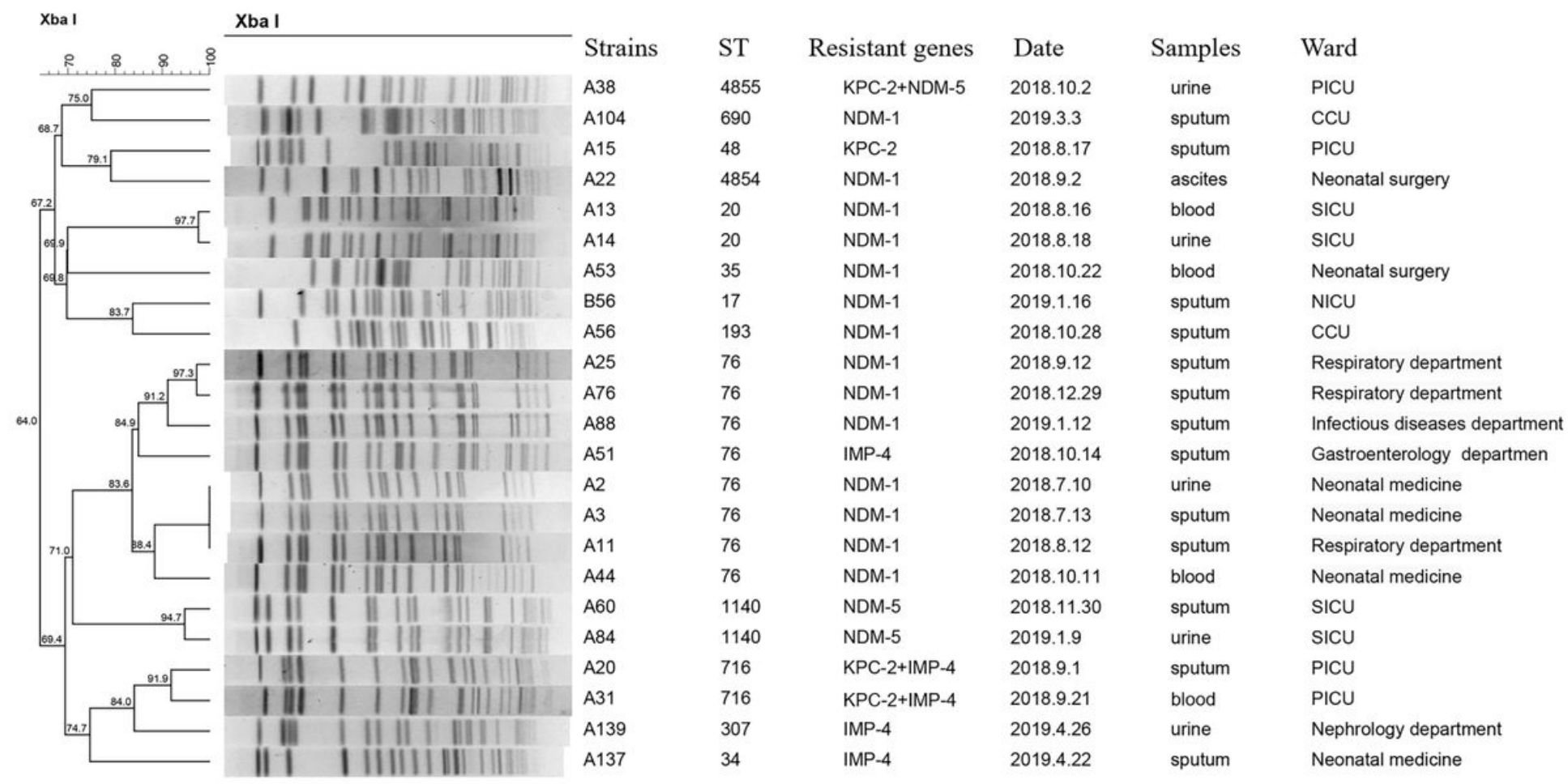

Figure 3

Dendrogram of PFGE profiles of 23 CRKP non-ST11 strains. The UPGMA algorithm was performed to construct dendrogram based on the dice similarity coefficient. Strains were classified as the same clone cluster when their dice similarity index was $\geq 80 \%$. 